\title{
Esophageal Subepithelial Lesion in a Patient with Painful Swallowing
}

Yoon Jin Choi

Division of Gastroenterology, Department of Internal Medicine, Severance Hospital, Yonsei University College of Medicine, Seoul, Korea

Question: A 41-year-old female patient visited the clinic complaining of non-progressive dysphagia, odynophagia, and postprandial epigastric pain lasting approximately 1 month. Otherwise, she was in good health and reported no other symptoms, including weight loss, fever or productive cough.

Esophagogastroduodenoscopy showed an about 2.0-cm sized subepithelial lesion at $25 \sim 27 \mathrm{~cm}$ from the incisor teeth (Fig. 1A). This lesion was accompanied by a linear ulcer on the surface (Fig. 1B). Furthermore, chest CT showed a protruding luminal lesion in the medial wall of the mid-esophagus (Fig. 2A) and necrotic lymphadenopathy in the subcarinal area, which compressed the esophageal lumen (Fig. 2B).
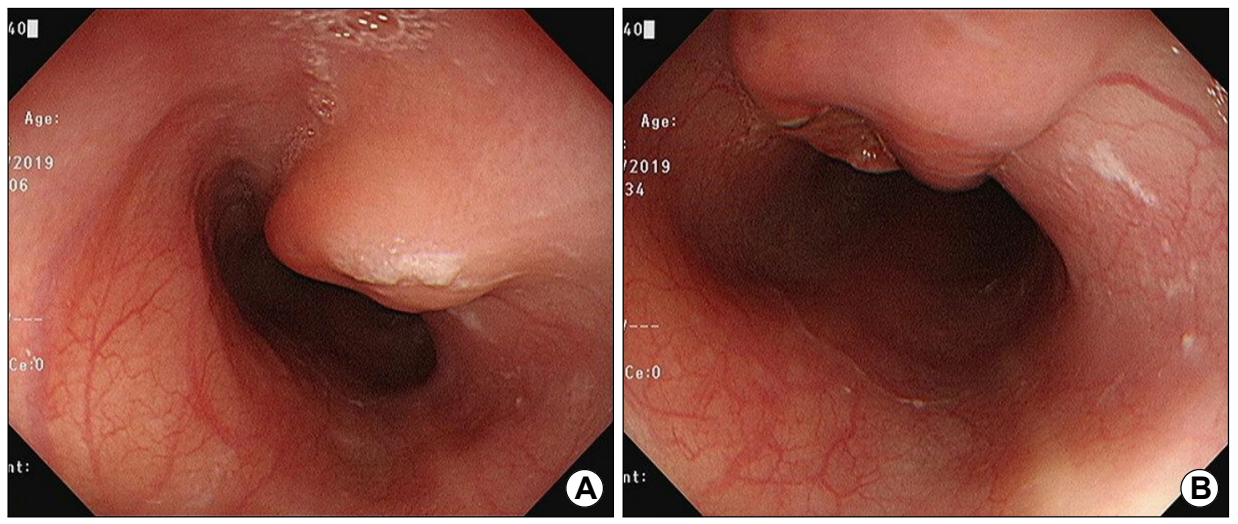

Fig. 1. Endoscopic findings showing (A) a 2.0-cm-sized subepithelial lesion on the mid-esophagus, with (B) ulceration on the surface.
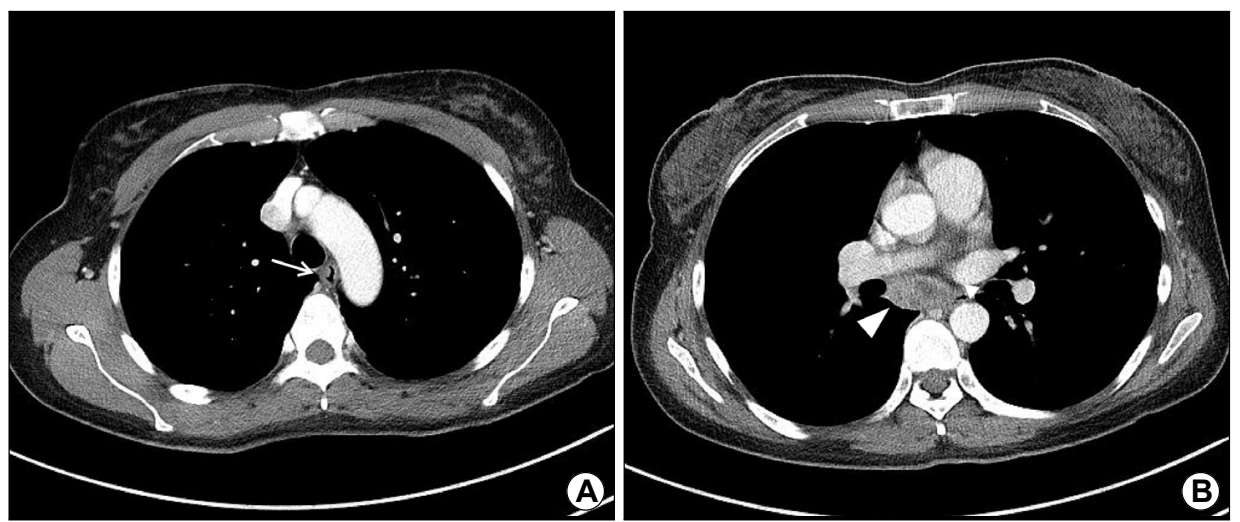

Fig. 2. Initial chest CT findings showing (A) a protruding luminal lesion on the mediolateral wall of the mid-esophagus (arrow) and (B) necrotic lymph node in the subcarinal area (arrowheads).

Received: January 15, 2020 Revised: January 28, 2020 Accepted: January 29, 2020

Corresponding author: Yoon Jin Choi

Division of Gastroenterology, Department of Internal Medicine, Severance Hospital,

Yonsei University College of Medicine, 50-1 Yonsei-ro, Seodaemun-gu, Seoul 03722, Korea

Tel: +82-2-2228-2264, Fax: +82-2-393-6884, E-mail: erica0007@gmail.co.kr

Copyright $\odot 2020$ Korean College of Helicobacter and Upper Gastrointestinal Research

(a) The Korean Journal of Helicobacter and Upper Gastrointestinal Research is an Open-Access Journal. All articles are distributed under the terms of the Creative Commons Attribution Non-Commercial License (http:// creativecommons.org/licenses/by-nc/4.0) which permits unrestricted non-commercial use, distribution, and reproduction in any medium, provided the original work is properly cited. 

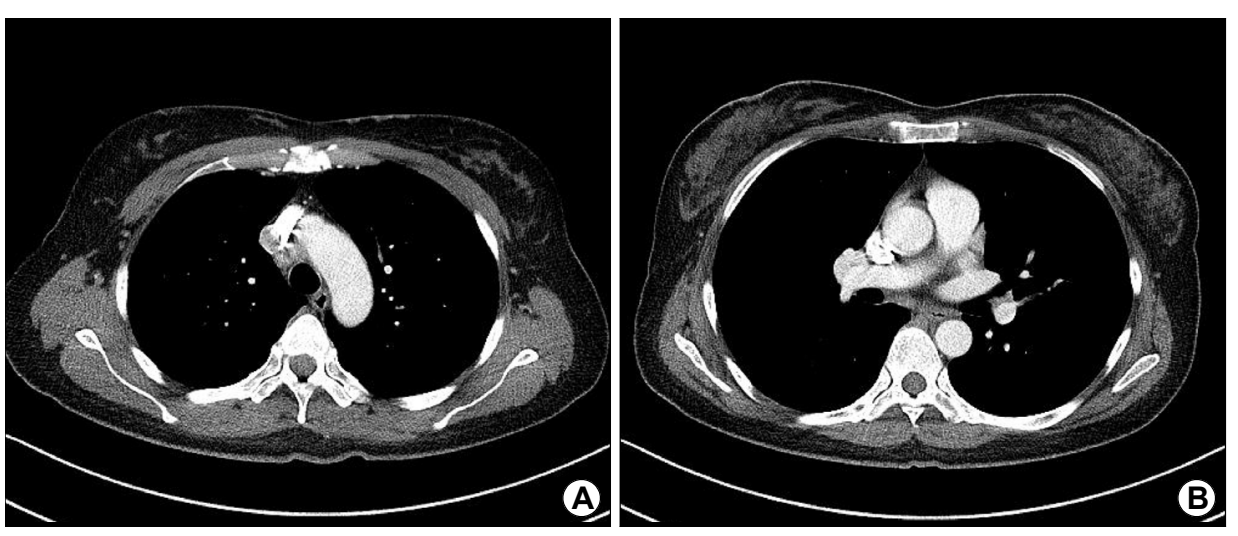

Fig. 3. Follow-up chest CT findings. (A, B) The previous protruding lesion in the mediolateral wall of the midesophagus and the necrotic lymph node in the subcarinal area are no longer visible.
What is the most likely diagnosis?

Answer: The endoscopic biopsy specimen obtained from ulcer of the lesion revealed vague granulomatous inflammation involving epithelioid histiocytes and lymphocytes in the stroma, pathologically suggesting tuberculosis. The result of acid-fast bacillus smear was negative, but the molecular nested PCR study for Mycobacterium tuberculosis was positive.

The patient underwent the first 2-month therapy for tuberculosis with isoniazid, pyrazinamide, rifampin, and ethambutol. Two months after anti-tuberculosis treatment, her symptoms of dysphagia disappeared, and odynophagia improved. Despite the doctor's recommendation, she refused repeated endoscopy. After another 4 months of medication, no symptoms or clinical signs remained. The repeated CT revealed no lesion in either esophagus (Fig. 3A) or subcarinal area (Fig. 3B).

Esophageal tuberculosis is a rare disease. Usually, it occurs as a result of direct extension of infection from mediastinal nodes, rarely the infection by spread from the lungs or bloodstream. Considering that Korea has a high prevalence of tuberculosis, the differential diagnosis of esophageal ulcerative, or mass-like lesions, practically subepithelial mass type at the mid-esophagus, should include esophageal tuberculosis. ${ }^{1}$ If esophageal tuberculosis is suspected, it can be diagnosed by endoscopic biopsies or postoperative pathology. However, multiple endoscopic biopsies often show only nonspecific chronic inflammation. If satisfactory findings are not found on the biopsy, esophageal tuberculosis should be differentiated by the detection of acid-resistant bacteria or by Mycobacterium tuberculosis $\mathrm{PCR}^{2}$ Although chest CT can help to find the primary site of tuberculosis, to determine lymphadenopathy or the presence of pulmonary esophageal fistulas, and to differentiate from malignant tumors of the esophagus, they cannot confirm esophageal tuberculosis. Lymphadenitis in the subcarinal area is one of the most common CT findings which patients with esophageal tuberculosis have. ${ }^{3}$ Since typical histological findings of this disease are rare in real practice, it is necessary to evaluate the clinical features, radiological and endoscopic findings, and response to anti-tuberculosis drugs to diagnose esophageal tuberculosis.

\section{CONFLICT OF INTEREST}

No potential conflict of interest relevant to this article was reported.

\section{ORCID}

Yoon Jin Choi ID https://orcid.org/0000-0002-1922-9388

\section{REFERENCES}

1. Patel N, Amarapurkar D, Agal S, et al. Gastrointestinal luminal tuberculosis: establishing the diagnosis. J Gastroenterol Hepatol 2004;19:1240-1246.

2. Cha S, Yeom DH. Sequential endoscopic findings of a tuberculous esophageal ulcer that developed into a traction diverticulum. Korean J Helicobacter Up Gastrointest Res 2020; 20:68-72.

3. Im JG, Song KS, Kang HS, et al. Mediastinal tuberculous lymphadenitis: CT manifestations. Radiology 1987;164:115-119. 